\title{
Zero-voltage nondegenerate parametric mode in Josephson tunnel junctions
}

\section{Pedersen, Niels Falsig}

Published in:

Journal of Applied Physics

Link to article, DOI:

$10.1063 / 1.322636$

Publication date:

1976

Document Version

Publisher's PDF, also known as Version of record

Link back to DTU Orbit

Citation (APA):

Pedersen, N. F. (1976). Zero-voltage nondegenerate parametric mode in Josephson tunnel junctions. Journal of Applied Physics, 47(2), 696-699. https://doi.org/10.1063/1.322636

\section{General rights}

Copyright and moral rights for the publications made accessible in the public portal are retained by the authors and/or other copyright owners and it is a condition of accessing publications that users recognise and abide by the legal requirements associated with these rights.

- Users may download and print one copy of any publication from the public portal for the purpose of private study or research.

- You may not further distribute the material or use it for any profit-making activity or commercial gain

- You may freely distribute the URL identifying the publication in the public portal

If you believe that this document breaches copyright please contact us providing details, and we will remove access to the work immediately and investigate your claim 


\title{
Zero-voltage nondegenerate parametric mode in Josephson tunnel junctions
}

\author{
N. F. Pedersen \\ Physics Laboratory I, The Technical University of Denmark, DK-2800 Lyngby, Denmark \\ (Received 8 April 1975)
}

\begin{abstract}
A new parametric mode in a Josephson tunnel junction biased in the zero-voltage mode is suggested. It is a nondegenerate parametric excitation where the junction plasma resonance represents the input circuit, and a junction geometrical resonance represents the idler circuit. This nondegenerate mode has been observed in analog experiments. In a real junction the realization of this mode of operation depends on the coupling between the plasma resonance and the geometrical resonance, and it is argued that without an external dc magnetic field the even geometrical resonances are most favorable for such a coupling.
\end{abstract}

PACS numbers: 74.30.M, 84.30.D, 85.25.

\section{INTRODUCTION}

A number of authors $\mathrm{s}^{1-10}$ have investigated parametric effects in Josephson junctions, the underlying reason being its potential as a high-frequency nonlinear element. Briefly, two different schemes for parametric effects have been considered: in one the junction is biased at a finite voltage $\mathrm{e}^{1-5}$ and the Josephson oscillation is used as the pump source, in the other scheme the junction is biased at zero voltage $e^{6-10}$ with a bias current $I_{\mathrm{dc}}<I_{0}$ ( $I_{0}$ is the maximum supercurrent), and an external pump is used. For the latter case the singly ${ }^{6}$ and the doubly ${ }^{7}$ degenerate modes have been discussed earlier. In the singly degenerate mode the pump frequency is at twice the junction plasma frequency; this mode has been observed indirectly in tunnel junction experiments at $\mathrm{X}$-band frequencies. ${ }^{8,9}$ In the doubly degenerate mode the pump frequency is close to the plasma frequency itself. An amplifier based on this principle, where the nonlinear element was an array of superconducting microbridges, has been reported recently. ${ }^{10}$ In this paper a zero-voltage nondegenerate parametric mode employing the junction plasma resonance $\omega_{p}$ as the input circuit and the junction geometrical resonance $\omega_{R}$ as the idler circuit is discussed. The external pump is at the frequency $\omega_{\text {pump }}=\omega_{p}+\omega_{R}$. In the derivation, an admittance matrix approach similar to the one normally used in connection with varactor diode amplifiers will be used.

The paper is organized along the following lines: In Sec. II the zero-voltage nondegenerate parametric mode is derived; in Sec. III the result is compared to analog experiments; and in Sec. IV the possibility for observation of the effect in a real junction experiment is discussed.

\section{THE ZERO-VOLTAGE NONDEGENERATE PARAMETRIC MODE}

The nondegenerate parametric mode behaves differently from the singly degenerate mode with respect to two important properties. First, for the singly degenerate a mplifier $\left(\omega_{\text {pump }} \sim 2 \omega_{\text {sigal }}\right)$ the gain depends on the relative phase between the pump and the signal, ${ }^{11}$ whereas for the nondegenerate amplifier the gain is independent of the relative phases. Second, the two modes are different with respect to noise properties, ${ }^{11}$ and which one is preferable depends on the particular application. The singly degenerate amplifier ${ }^{6}$ includes one resonant circuit, tuned to the signal frequency $\omega_{s}$. The nondegenerate amplifier, to be discussed here (the lower sideband up-converter ${ }^{11}$ ) includes a second resonant circuit, the idler circuit, with resonance frequency $\omega_{i}>\omega_{s}$.

A junction equivalent circuit model with two resonance frequencies is shown in Fig. 1. It consists of a Josephson element satisfying the usual Josephson equations

$$
\begin{aligned}
& I=I_{0} \sin \phi, \\
& \frac{d \phi}{d t}=\frac{2 e}{\hbar} V,
\end{aligned}
$$

where $\phi$ is the phase difference, $V$ is the voltage across the junction, and $I_{0}$ is the maximum supercurrent. The Josephson element is shunted by a constant resistor $R$, a capacitor $C$, and by a series resonant circuit with inductance $L_{0}$, resistance $r$, and capacitance $C$. In a thin-film tunnel junction the series inductance $L_{0}$ arises from the imaginary part of the film surface impedance, which together with the capacitance $C$, gives rise to the geometrical resonance, $\omega_{R}$.

In the following the real part of the surface impedance $r$ will be neglected since it can be made small ${ }^{12}$ by lowering the temperature. When the junction is biased in the zero-voltage mode $\left(I_{\mathrm{dc}}<I_{0}\right)$, the Josephson element may be represented by an ideal inductance ${ }^{7} L_{1}$ defined by

$$
\frac{1}{L_{1}}=\frac{2 e I_{0}}{\hbar} \cos \phi_{0}
$$

as shown in Fig. 1. Here $\sin \phi_{0}=I_{\mathrm{dc}} / I_{0}$.

The assumed equivalent circuit is only an approximate representation of the real tunnel junction. The

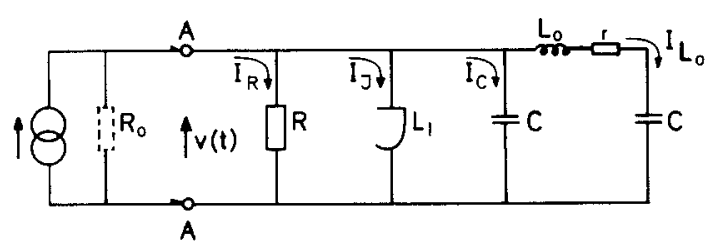

FIG. 1. Josephson junction model with a series resonant circuit. 
approximations inherent in the circuit model will be discussed further in Sec. IV.

The two resonance frequencies of Fig. 1 are given by

$$
\begin{aligned}
& \omega_{1}^{2}=\frac{2 L_{1}+L_{0}}{2 L_{1} L_{0} C}-\frac{\left(4 L_{1}^{2}+L_{0}^{2}\right)^{1 / 2}}{2 L_{1} L_{0} C}, \\
& \omega_{2}^{2}=\frac{2 L_{1}+L_{0}}{2 L_{1} L_{0} C}+\frac{\left(4 L_{1}^{2}+L_{0}^{2}\right)^{1 / 2}}{2 L_{1} L_{0} C} .
\end{aligned}
$$

In the limit $L_{0} \rightarrow 0$ the resonance frequency is the plasma frequency $\omega_{p}=\left(2 C L_{1}\right)^{-1 / 2}$, and in the limit $L_{1} \rightarrow \infty$ the resonance frequency is the geometrical resonance frequency $\omega_{R}=\left(2 / L_{0} C\right)^{1 / 2}$. Assuming $L_{0} \ll L_{1}$, that is, $\omega_{R}$ $\gg \omega_{p}$, we find that the resonance requencies of Eq. (4) are close to $\omega_{p}$ and $\omega_{R}$. An expansion of Eq. (4) for $\omega_{p} / \omega_{R} \ll 1$ gives

$$
\begin{aligned}
& \omega_{1} \sim \omega_{p}\left(1-\frac{1}{2} \frac{\omega_{p}^{2}}{\omega_{R}^{2}}\right), \\
& \omega_{2} \sim \omega_{R}\left(1+\frac{1}{2} \frac{\omega_{p}^{2}}{\omega_{R}^{2}}\right) .
\end{aligned}
$$

For the circuit, Fig. 1, we now assume that the junction voltage has the for $\mathrm{m}^{13}$

$$
V(t)=V_{p} \cos \left(\omega_{\text {pump }} t\right)+\operatorname{Re}\left[V_{1} \exp \left(j \omega_{1} t\right)+V_{2} \exp \left(j \omega_{2} t\right)\right],
$$

where

$$
V_{p} \gg\left|V_{1}\right|,\left|V_{2}\right|
$$

and

$$
\omega_{\text {pump }}=\omega_{1}+\omega_{2} \text {. }
$$

The notation in Eq. (6) implies that the phases of $V_{1}$ and $V_{2}$ are measured relative to the phase of the pump signal. By integrating Eq. (2), using Eq. (6), and inserting $\phi$ in Eq. (1), a first-order expansion in the small quantities $V_{1}$ and $V_{2}$ by means of the FourierBessel theorem ${ }^{14}$ gives components of the supercurrent $I_{1 J}$ and $I_{2 J}^{*}$ at frequencies $\omega_{1}$ and $\omega_{2}$, respectively,

$$
\left\{\begin{array}{l}
I_{1 J} \\
I_{2 J}^{*}
\end{array}\right\}=\left\{\begin{array}{ll}
Y_{11} & Y_{12}^{*} \\
Y_{21} & Y_{22}^{*}
\end{array}\right\}\left\{\begin{array}{l}
V_{1} \\
V_{2}^{*}
\end{array}\right\},
$$

where

$$
\begin{array}{ll}
Y_{11}=\frac{1}{j} \frac{2 e I_{0} J_{0}(\alpha)}{\hbar \omega_{1}} \cos \phi_{0}, & Y_{12}^{*}=-\frac{2 e I_{0} J_{1}(\alpha)}{\hbar \omega_{2}} \sin \phi_{0}, \\
Y_{21}=\frac{2 e I_{0} J_{1}(\alpha)}{\hbar \omega_{1}} \sin \phi_{0}, & Y_{22}^{*}=-\frac{1}{j} \frac{2 e I_{0} J_{0}(\alpha)}{\hbar \omega_{2}} \cos \phi_{0} .
\end{array}
$$

Here $J_{n}(\alpha)$ is the $n$ th-order Bessel function of argument $\alpha=2 e V_{p} / \hbar \omega_{\text {pump }}$. The elements $Y_{11}$ and $Y_{22}^{*}$ corresponds to the inductor $L_{1}$ in Fig. 1 and $Y_{12}^{*}$ and $Y_{21}$ represents lower sideband mixing products at frequencies $\omega_{\text {pump }}$ $-\omega_{2}=\omega_{1}$ and $\omega_{\text {pump }}-\omega_{1}=\omega_{2}$, respectively. It is observed $^{7}$ from Eq. (9) that the off-diagonal coupling strength is a function of the pump amplitude $V_{p}$.

Referring the currents to the outside of the junction (left of AA in Fig. 1) we have

$$
l=I_{R}+I_{J}+I_{C}+I_{L} \text {, }
$$

where $I_{R}, I_{C}$, and $I_{L_{0}}$ contributes only to the diagonal elements when added to $I_{J}$, Eq. (9). Further, since the circuit is resonant at frequencies $\omega_{1}$ and $\omega_{2}$ the imaginary part of the diagonal elements vanishes and we obtain for the total current to the junction $I_{1}$ and $I_{2}^{*}$ at frequencies $\omega_{1}$ and $\omega_{2}$

$$
\left\{\begin{array}{l}
I_{1} \\
I_{2}^{*}
\end{array}\right\}=\left\{\begin{array}{ll}
1 / R & Y_{12}^{*} \\
Y_{21} & 1 / R
\end{array}\right\}\left\{\begin{array}{c}
V_{1} \\
V_{2}^{*}
\end{array}\right\},
$$

where $Y_{12}^{*}$ and $Y_{21}$ are given by Eq. (9).

If there is no external source at the frequency $\omega_{2}$ we obtain

$$
I_{2}^{*}=-V_{2}^{*} / R_{0}
$$

where $R_{0}$ is the waveguide impedance (or free-space impedance) at the frequency $\omega_{2}$. Combining Eqs. (11) and (12) we find the current $I_{1}$ at the frequency $\omega_{1}$ to be

$$
I_{1}=\frac{V_{1}}{R}-\frac{Y_{12}^{*} Y_{21}}{1 / R+1 / R_{0}} V_{1} \text {. }
$$

Since $R_{0}$ is typically much greater than $R$ we may substitute $1 / R$ for $1 / R+1 / R_{0}$ and we find for the input admittance at frequency $\omega_{1}$

$$
Y_{\text {in }}\left(\omega_{1}\right)=\frac{I_{1}}{V_{1}}=\frac{1}{R}\left(1-\frac{\left[\left(2 e I_{0} / \hbar\right) \sin \phi_{0} J_{1}(\alpha) R\right]^{2}}{\omega_{1} \omega_{2}}\right),
$$

where $Y_{12}^{*}$ and $Y_{21}$ have been inserted from Eq. (9). Inserting ${ }^{6} Q=\omega_{p} R C, J_{1}(\alpha) \sim \frac{1}{2} \alpha$, and using $\omega_{1} \sim \omega_{p}$ and $\omega_{2} \sim \omega_{R}$ [Eq. (5)] we may approximate Eq. (14);

$$
Y_{\mathrm{tn}}\left(\omega_{p}\right) \approx \frac{1}{R}\left[1-\frac{\omega_{p}}{\omega_{R}}\left(\frac{\alpha \tan \phi_{0}}{2 / Q}\right)^{2}\right] .
$$

The input admittance at the plasma resonance becomes negative when the threshold condition

$$
\alpha \tan \phi_{0}>\left(\frac{\omega_{R}}{\omega_{p}}\right)^{1 / 2} \frac{2}{Q}
$$

is satisfied.

For the singly degenerate mode $\left(\omega_{\text {pump }} \approx 2 \omega_{p}\right)$ the input admittance at the plasma frequency may be shown to be

$$
Y_{\text {Ia }}\left(\omega_{p}\right) \approx \frac{1}{R}\left(1-\frac{\alpha \tan \phi_{0}}{2 / Q} \exp \left(-j \theta_{s}\right)\right)
$$

with a threshold condition ${ }^{6}$ for parametric oscillations

$$
\alpha \tan \phi_{0}>2 / Q
$$

here $\theta_{s}$ is the phase of the signal voltage relative to the pump voltage.

When comparing Eqs. (16) and (18) we note that the threshold pump power has been raised in the nondegenerate mode; however, as can be seen from Eqs. (15) and (17) the phase dependence has disaapeared. Also the noise temperature of the nondegenerate mode has been reduced compared to the singly degenerate mode since it is proportional to $\omega_{p} / \omega_{R} .{ }^{11}$ It is noted that the

formalism used here is the same as the one commonly used for varactor diode parametric amplifiers. Hence, once the negative input admittance is derived, formulas for gain, bandwidth, noise temperature, etc., may be found in standard textbooks ${ }^{11}$ with suitable substitutions ${ }^{15}$ changing the nonlinear series capacitance of the varactor to a nonlinear parallel inductance. 


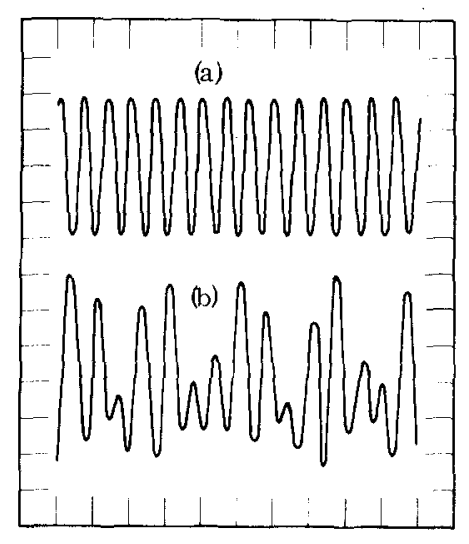

FIG. 2. Voltage waveforms (a) just below threshold, (b) just above threshold. Scale: horizontal axis, $50 \mu \mathrm{s} / \mathrm{div}$; vertical axis, $50 \mathrm{mV} / \mathrm{div} . f_{\text {pump }}=34.4 \mathrm{kHz}, f_{p}=9.4 \mathrm{kHz}$, and $f_{R}=25 \mathrm{kHz}$. Circuit parameters are in the text.

\section{ANALOG EXPERIMENTS}

The preceding calculations were compared to corresponding analog measurements. The analog has been described elsewher $\mathrm{e}^{16}$ and has an equivalent diagram similar to Fig. 1 with the following parameters: $I_{0}$ $=1 \mathrm{~mA}, k=2 e / h=200 \mathrm{kHz} / \mathrm{V}, R=500 \Omega$, and $C=100$ $\mathrm{nF}$. The plasma frequency $\omega_{p} / 2 \pi$ was adjusted to 9.4 $\mathrm{kHz}\left(I_{\mathrm{dc}}=0.6 \mathrm{~mA}\right)$ and $L_{0}$ was chosen to give $\omega_{R} / 2 \pi=25$ $\mathrm{kHz}$. The analog experiment proceeded in the following way. The two resonance frequencies $\omega_{1}\left(\approx \omega_{p}\right)$ and $\omega_{2}$ $\left(\approx \omega_{R}\right)$ were measured in the small-signal limit; a pump signal was applied at the frequency $\omega_{1}+\omega_{2}$ and the amplitude was gradually increased while the voltage waveform was observed on an oscilloscope. At a certain threshold pump voltage $V_{\mathrm{rf}}^{\mathrm{crt}}$, the voltage waveform suddenly changed and the frequencies $\omega_{1}$ and $\omega_{2}$ appeared with large amplitude as seen in Fig. 2(b). Figure 2(a) shows the voltage waveform just below the threshold rf voltage, it contains only the pump frequency (and some higher harmonics). Figure 3 shows the quantity $\alpha_{c}=k V_{\mathrm{rf}}^{\mathrm{ait}} / f_{\mathrm{pump}}$ as a function of the pump frequency. Curve (a) of Fig. 3 shows the threshold curve for the nondegenerate parametric excitation when pumped with a frequency in the vicinity of $\omega_{p}+\omega_{R}$ and curve (b) of Fig. 3 shows the threshold curve for the singly degenerate parametric excitation when the pump frequency is twice the plasma frequency. ${ }^{6}$ Taking the total circuit losses into account, the threshold values of Fig. 3 are in agreement with the derived ones [Eqs. (16) and (18)] within the experimental uncertainty.

In addition, different types of frequency conversion were investigated. The voltage components at the different frequencies were measured by means of a spectrum analyzer. For example if one $\mathrm{rf}$ biased the analog just below the threshold curve at a frequency $\omega_{p}+\omega_{R}$ and applied a small signal at $\omega_{p}$, an amplified signal at $\omega_{R}$ could be observed in general agreement with the Manley-Rowe relations. ${ }^{11}$

\section{POSSIBILITIES FOR A REAL JUNCTION EXPERIMENT}

Both the singly and doubly degenerate parametric modes have recently been observed experimentally. ${ }^{8-10}$ Here we discuss, at least qualitatively, the possibilities for an experiment on the nondegenerate parametric mode. The approximative model assumed in Sec. II is a too simplified model for the situation in a real junction. In a real junction the plasma mode and the geometrical mode have different electromagnetic field configurations and, hence, a lumped circuit model as in Fig. 1 is insufficient However, the important requirement for the theory to hold is that a coupling between the plasma mode and the geometrical mode exists. Theoretically, this coupling could be calculated by setting up Maxwell's equations and calculating the spatial overlap between the two modes. However, this would require a computer calculation based on a distributed junction model. Experimentally, only in plasma resonance experiments ${ }^{17}$ such a coupling has been reported.

That a coupling between the plasma mode and the geometrical mode exists may be understood from simple intuitive arguments.

The plasma mode is assumed to have a uniform longitudinal (i.e., transverse to the barrier) electric field. The geometrical mode has a spatially varying longitudinal component of the electric field. For the odd modes the electric field at the two junction edges point in opposite directions. For the even modes, however, the electric field at the two edges point in the same direction. Thus these modes have a spatial overlap (depending on the standing wave ratio) to the uniform plasma mode and seems more favorable for a coupling. These qualitative arguments may be further supported by comparison to the so-called self-induced steps. ${ }^{18}$ In this case the (spatially uniform electric field due to the Josephson voltage couples to the even geometrical modes. In any case, the spatial overlap and, hence, the coupling may be improved by an applied dc magnetic field, since the spatial variation of the plasma mode electric field is magnetic field dependent, whereas the geometrical mode field configuration is not.

Finally, by controlled fabrication of a tunnel junction, $\omega_{b}$ and $\omega_{R}$ may be chosen independently since they depend partly on different junction parameters.

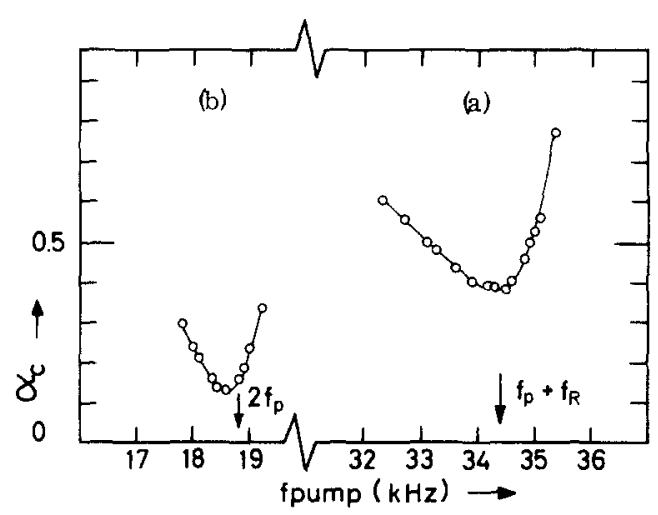

FIG. 3, Threshold curves for 6) the nondegenerate mode and (b) the singly degenerate mode, as a function of the pump frequency. $f_{p}=9.4 \mathrm{kHz}$ and $f_{R}=25 \mathrm{kHz}$. Ordinate: $\alpha_{c}=k V_{\mathrm{r} f}^{\mathrm{crt}} / f_{\mathrm{pumo}}$. Circuit parameters are in the text. 


\section{CONCLUSION}

A nondegenerate parametric element (lower sideband up-converter), using the junction plasma resonance and the junction geometrical resonance as signal and idler circuits, respectively, has been suggested. The element has been investigated by means of a Josephson junction analog, and agreement between the calculations and the analog results was found.

\section{ACKNOWLEDGMENT}

Stimulating discussions with M.R. Samuelsen, O. Hoffmann Soer ensen, C. K. Bak, E. Kollberg, and O. Nilsson are gratefully acknowledged.

${ }^{1}$ P. Russer, Arch. Elektrischen Übertragung 23, 417 (1969).

${ }^{2}$ H. Kanter and A. H. Silver, Appl. Phys. Lett. 19, 515 (1971).

${ }^{3}$ H. Zimmer, Appl. Phys. Lett. 10, 193 (1967).

${ }^{4}$ A. N. Vystavkin, V.N. Gubankov, G. F. Leschenko, K. K. Likharev, and V.V. Migulin, Radio Eng. Electron. Phys. 15, 2121 (1970).

${ }^{5}$ A. N. Vystavkin, V.N. Gubankov, L.S. Kuzmin, K. K. Likharev, V.V. Migulin, and V.K. Semenov, Rev. Phys. Appl. 9, 79 (1974)

${ }^{6}$ N.F. Pedersen, M.R. Samuelsen, and K. Saermark, J. Appl. Phys. 44, 5120 (1973).

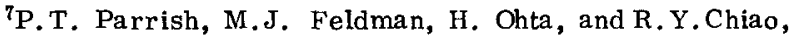
Rev. Phys. Appl. 9, 229 (1974).

${ }^{8}$ C. K. Bak, B. Kofoed, N. F. Pedersen, and K. Saermark, Proceedings of the applied Superconductivity Conference, 1974, Oakbrook, IIl. (unpublished); J. Appl. Phys. 46, 886 (1975).

${ }^{9}$ A. J. Dahm and D.N. Langenberg, J. Low Temp. Phys. (to be published).

${ }^{10}$ P. T. Parrish and R. Y. Chiao, Appl. Phys. Lett. 25, 627 (1974).

${ }^{11}$ P. PennfieId and R. P. Rafuse, Varactor Applications (MIT Press, Cambridge, Mass., 1962).

${ }^{12}$ O. H. Soerensen, T. F. Finnegan, and N. F. Pedersen, Appl. Phys. Lett. 22, 129 (1973).

${ }^{13}$ If the junction is considered ac-cur rent driven, harmonics of $\omega_{\text {pump }}, \omega_{1}$ and $\omega_{2}$ will appear in the voltage. These frequency components may be retained in the calculations (Ref. 11). However, unless the junction has a resonance at one of these frequencies only minor quantitative changes will occur in the first-order calculations discussed here.

${ }^{14} \mathrm{M}$. Abramowitz and I. A. Stegun, Handbook of Mathematical Functions (Dover, New York, 1965).

${ }^{15}$ The substitutions are $C(t) \rightarrow 1 / L(t), r \rightarrow 1 / R$ : and $V \rightarrow I$.

${ }^{16}$ C. K. Bak and N. F. Pedersen, Appl. Phys. Lett. 22, 149 (1973).

${ }^{17}$ N. F. Pedersen, T. F. Finnegan, and D. N. Langenberg, Proceedings of the Thirteenth International Conference on Low Temperature Physics (Plenum, New York, 1974), Vol. III, p. 268; Phys. Rev. B 6, 4151 (1972).

${ }^{18} \mathrm{C} . \mathrm{T}$. Chen and D. N. Langenberg, Proceedings of the Thirteenth International Conference on Low Temperature Physics (Plenum, New York, 1974), Vol. III, p. 289. 